\title{
ABSORCIÓN DE FÁRMACOS POR VÍA TÓPICA. PAPEL DE LA CONJUNTIVA
}

\section{OCULAR DRUG ABSORPTION BY TOPICAL ROUTE. ROLE OF CONJUNCTIVA}

\author{
ANDRÉS-GUERRERO V ${ }^{1}$, HERRERO-VANRELL R ${ }^{2}$
}

La penetración de fármacos al interior del ojo tras su administración por vía tópica ocular es uno de los retos más interesantes a los que se enfrentan las ciencias farmacéuticas. El objetivo del científico es, en este caso, conseguir atravesar las barreras protectoras del ojo sin dañar las estructuras oculares.

A diferencia de otros, los epitelios de la superficie ocular no son zonas preparadas para la absorción, por lo que el tiempo de residencia de una formulación convencional se reduce a menos de dos minutos. A esto hay que añadir las pérdidas generadas por la absorción sistémica del fármaco a través de la conjuntiva y el conducto nasolacrimal. Se estima que el porcentaje de sustancia activa capaz de alcanzar el segmento anterior del ojo está comprendido entre el $1-5 \%$ de la dosis total administrada. Por esta razón, aunque el fármaco sea capaz de llegar a humor acuoso su difusión al segmento posterior resulta insignificante, en la mayoría de los casos.

La penetración de los fármacos cuando se administran por vía tópica ocular se puede llevar a cabo a través de la córnea (vía transcorneal) o a través de la conjuntiva y esclera (vía conjuntival/escleral). Se sabe que la mayoría de las sustancias activas que se emplean por vía tópica se absorben empleando la ruta transcorneal. Este hecho explica que, durante mucho tiempo, se pensara en la conjuntiva únicamente como una vía de eliminación. Hoy en día se sabe que hay sustancias capaces de atravesar la conjuntiva llegando a alcanzar, en ciertas ocasiones, el segmento posterior (1).

En general, el epitelio de la córnea permite el paso de sustancias activas hidrofóbicas de pequeño tamaño a través de las células epiteliales por difusión (ruta transcelular) y el de sustancias hidrofílicas entre los espacios de las mismas en lo que se conoce como ruta paracelular (2).

La conjuntiva es una membrana mucosa que recubre aproximadamente el $80 \%$ de la superficie ocular. Está formada por una porción bulbar que cubre la parte anterior del globo, excepto la córnea, y dos porciones palpebrales en las caras posteriores de los párpados superior e inferior. El epitelio conjuntival está compuesto por una serie de capas de células epiteliales estratificadas (de 5 a 15 capas) y se encuentra recubierto por microvellosidades. La conjuntiva bulbar está recubierta por la película lacrimal y contribuye a la formación de esta última mediante la secreción de electrolitos, mucus y glicoproteínas.

Al igual que la córnea, el paso a través de la conjuntiva se puede llevar a cabo por la vía transcelular o paracelular (3). Si nos centramos en las propiedades generales de las sustancias activas, el tamaño molecular es el factor limitante de la ruta paracelular tanto de la córnea como de la conjuntiva. Las rutas paracelulares fueron descritas hace algunos años en conejos empleando polietilénglicoles de distintos pesos moleculares (de 200 a 1000) (4). Se seleccionaron dichos agentes ya que sus características resultan comunes a péptidos y oligonucleótidos (hidrofilia, capacidad de formar puentes de hidrógeno y tamaño molecular). En este estudio, la conjuntiva resultó ser mucho más permeable y menos restrictiva que la córnea. El tamaño molecu-

\footnotetext{
Departamento de Farmacia y Tecnología Farmacéutica. Facultad de Farmacia. Universidad Complutense. Madrid. España.

1 Licenciada en Farmacia.

E-mail: vandres@farm.ucm.es

2 Doctora en Farmacia. Profesora Titular de Universidad.

E-mail: rociohv@farm.ucm.es
} 
lar de los espacios intercelulares en la conjuntiva es, aproximadamente, el doble que los de la córnea $(5,5$ $\mathrm{nm})$ y la densidad de poro 16 veces superior $\left(1,9 \times 10^{8}\right.$ poros $\left./ \mathrm{cm}^{2}\right)$ (4). Por otro lado, las células epiteliales de la conjuntiva presentan las mismas uniones intercelulares que caracterizan al epitelio corneal conocidas como «tight junctions», aunque la conjuntiva es más permeable al paso de moléculas hidrofílicas. Además se estima que el espacio paracelular de la conjuntiva es más de doscientas veces superior al de la córnea.

En un primer momento, las investigaciones en torno a la absorción conjuntival se han centrado en potenciar la vía transcelular o paracelular. Para potenciar la vía transcelular se ha acudido al aumento de la lipofilia del fármaco utilizando profármacos o análogos de la sustancia activa, mientras que la vía paracelular se ha potenciado mediante el uso de promotores que favorecen la apertura de las uniones intercelulares.

$\mathrm{Al}$ constatar que el paso de sustancias a través de la conjuntiva se puede producir además por transporte activo, por endocitosis o a través del espacio subconjuntival (mediante inyecciones, implantes o por iontoforesis) se han abierto nuevas vías de investigación en este campo.

Hay evidencias de la existencia de transporte activo conjuntival similar al que tiene lugar en el intestino, aunque en el primer caso es más restrictivo (3). Un ejemplo de este tipo es el transportador de sodio monocarboxilato que se ha propuesto para fármacos aniónicos como el cromolin, el flurbiprofeno y el diclofenaco empleados para el tratamiento de distintos tipos de conjuntivitis. Los mecanismos de transporte activo de iones $\mathrm{Cl}-, \mathrm{Na}+\mathrm{y} \mathrm{K}+$ contribuyen al paso de líquido a través del epitelio también pueden aprovecharse para promover la absorción de fármacos a través de la conjuntiva. Así, mientras que la secreción de Cl- se acompaña de secreción de líquido, la absorción de $\mathrm{Na}+$ lo hace con la absorción de líquido. De esta forma, si la zona apical de la conjuntiva se expone a nutrientes que inducen la absorción de $\mathrm{Na}$ + (como los aminoácidos) se puede favorecer, al mismo tiempo, la absorción de líquido. Si este proceso fisiológico se une a la apertura de las uniones intercelulares mediante otros mecanismos, se favorecería la absorción de solutos hidrofílicos a través de la membrana.

El proceso de endocitosis por las células epiteliales de la conjuntiva también resulta una alternativa interesante y ha sido ya descrita para nanopartículas de tamaño $100 \mathrm{~nm}$ preparadas a partir del ácido poliláctico-co-glicólico (PLAGA) (5). Aunque este fenómeno se encuentra aún en estudio, hay un interés especial en las investigaciones dirigidas en este sentido.

La vía subconjuntival también se presenta como alternativa a las inyecciones intravítreas que resultan más agresivas para el paciente. Esta vía persigue incrementar la concentración intraocular de fármaco disminuyendo su frecuencia de administración. Con este fin se han desarrollado implantes subconjuntivales (6) y nano- y micropartículas (7). Estas últimas dieron lugar a concentraciones de sustancia activa en el vítreo y en distintas zonas de la retina.

La iontoforesis es una técnica según la cual se consigue que los fármacos penetren la membrana de forma ionizada mediante la aplicación de una corriente eléctrica, por lo que se restringe a sustancias activas capaces de ionizarse. La corriente eléctrica favorece el paso del fármaco a través de la barrera celular. Esta técnica permite variar la densidad de corriente y el tiempo de aplicación de la misma consiguiendo distintos efectos. La iontoforesis se puede aplicar tanto en la córnea (iontoforesis transcorneal) como en la conjuntiva y esclera (iontoforesis transconjuntival/escleral) (8) y ha permitido incrementar las concentraciones de fármaco en vítreo y retina.

Otro dato interesante es el papel de la conjuntiva como ruta de absorción de proteínas o péptidos cuyo paso a través de la córnea está limitado. Sin embargo, no hay que olvidar que la actividad enzimática de esta zona puede degradar significativamente la sustancia activa tal y como ha sido descrito para insulina y sustancia P (9). Este fenómeno se puede intentar solventar añadiendo, por ejemplo, agentes inhibidores de proteasas a la formulación.

Hoy en día las rutas de acceso que ofrece la conjuntiva están en estudio (ruta paracelular, transcelular, transporte activo o endocitosis) y se están investigando distintas estrategias para favorecer la penetración de sustancias activas. La iontoforesis transconjuntival se plantea como una técnica no invasiva y segura que puede mejorar el paso de sustancias hasta el vítreo y los tejidos de la retina. Sin embargo, los mecanismos de absorción de la conjuntiva no se encuentran tan descritos como los de la córnea. En el momento en que esto ocurra las rutas de acceso a través de esta vía serán utilizadas de forma más efectiva. 


\section{BIBLIOGRAFÍA}

1. Urtti A. Challenges and obstacles of ocular pharmacokinetics and drug delivery. Adv Drug Deliv Rev 2006; 58: 1131-1135.

2. Prausnithz MR, Noonan JS. Permeability of cornea, sclera and conjunctiva: a literature analysis for drug delivery to the eye. J Pharm Sci 1998; 87: 1479-1488.

3. Hosoya K, Lee VH, Kim KJ. Roles of the conjunctiva in ocular drug delivery: a review of conjunctival transport mechanisms and their regulation. Eur J Pharm Biopharm 2005; 60: 227-240.

4. Hämäläinen KM, Kananen K, Auriola S, Kontturi K, Urtti A. Characterization of paracellular and aqueous penetration routes in cornea, conjunctiva and sclera. Invest Ophthalmol Vis Sci 1997; 38: 627-634.

5. Qaddoumi MG, Ueda H, Yang J, Davda J, Labhasetwar V, Lee VH. The characteristics and mechanisms of uptake of
PLGA nanoparticles in rabbit conjunctival epithelial cell layers. Pharm Res 2004; 21: 641-648.

6. Wang $G$, Tucker IG, Roberts MS, Hirst $L W$. In vitro and in vivo evaluation in rabbits of a controlled release 5-fluorouracil subconjunctival implant based on poly $(D, L$-lactide-co-glycolide). Pharm Res 1996; 13: 1059-1064.

7. Amrite AC, Kompella UB. Size-dependent disposition of nanoparticles and microparticles following subconjunctival administration. J Pharm Pharmacol 2005; 57: 15551563.

8. Raiskup-Wolf F, Eljarrat-Binstock E, Rehák M, Domb A, Frucht-Pery J. Transcorneal and transscleral iontophoresis of the dexamethasone phosphate into the rabbit eye. $J$ Control Release 2007; 63: 360-368.

9. Sunkara G, Kompella UB. Membrane transport processes in the eye. In: Mitra AK. Drugs and the pharmaceutical science. Ophthalmic drug delivery systems. New York: Marcel Dekker, Inc.; 2003; 13-58. 